\title{
Symmetries, Traveling Wave Solutions, and Conservation Laws of a $(3+1)$-Dimensional Boussinesq Equation
}

\author{
Letlhogonolo Daddy Moleleki and Chaudry Masood Khalique \\ International Institute for Symmetry Analysis and Mathematical Modelling, Department of Mathematical Sciences, \\ North-West University, Mafikeng Campus, Private Bag X 2046, Mmabatho 2735, South Africa
}

Correspondence should be addressed to Chaudry Masood Khalique; masood.khalique@nwu.ac.za

Received 5 April 2014; Accepted 15 June 2014; Published 2 July 2014

Academic Editor: Xiao-Yan Gu

Copyright (C) 2014 L. D. Moleleki and C. M. Khalique. This is an open access article distributed under the Creative Commons Attribution License, which permits unrestricted use, distribution, and reproduction in any medium, provided the original work is properly cited.

We analyze the $(3+1)$-dimensional Boussinesq equation, which has applications in fluid mechanics. We find exact solutions of the $(3+1)$-dimensional Boussinesq equation by utilizing the Lie symmetry method along with the simplest equation method. The solutions obtained are traveling wave solutions. Moreover, we construct the conservation laws of the $(3+1)$-dimensional Boussinesq equation using the new conservation theorem, which is due to Ibragimov.

\section{Introduction}

It is well known that the $(1+1)$-dimensional Boussinesq equation [1],

$$
u_{t t}-u_{x x}-\left(u^{2}\right)_{x x}-u_{x x x x}=0
$$

describes the propagation of long waves on the surface of water with a small amplitude and plays a vital part in fluid mechanics [2]. It is completely integrable and admits multiple soliton solutions.

The $(2+1)$-dimensional Boussinesq equation

$$
u_{t t}-u_{x x}-u_{y y}-\alpha\left(u^{2}\right)_{x x}-u_{x x x x}=0,
$$

which describes the propagation of gravity waves on the surface of water, has been extensively studied by several authors (see, e.g., [3-7]).

The $(3+1)$-dimensional Boussinesq equation is given by

$$
u_{t t}-u_{x x}-u_{y y}-u_{z z}-\alpha\left(u^{2}\right)_{x x}-u_{x x x x}=0 .
$$

In [8], the author obtained one-periodic wave solution, two-periodic wave solution, and soliton solution for (3) by means of Hirota's bilinear method and the Riemann theta function. Wazwaz [5] employed a combination of Hirota's method and Hereman's method to formally study (3) and derived two soliton solutions of (3). Some other work concerning symmetries and exact solutions of some Boussinesq equations can be seen in [9-12].

In the last few decades several methods have appeared in the literature, which can be used to find exact solutions of nonlinear evolution equations (NLEEs). Some of these methods are the inverse scattering transform method [13], the Darboux transformation method [14], the sine-cosine method [15], Hirota's bilinear method [16], Jacobi elliptic function expansion method [17], Lie group analysis [18-20], and the exp-function expansion method [21].

In this paper we use Lie group method along with the simplest equation method $[22,23]$ to construct some exact solutions of (3). Furthermore, we employ the new conservation theorem due to Ibragimov [24] to derive conservation laws for (3).

Lie group method, which was developed by Sophus Lie (1842-1899) in the nineteenth century, is a systematic method that can be used to find solutions of nonlinear partial differential equations (PDEs). It is based upon the study of the invariance under one-parameter Lie group of point transformations $[18,19]$.

Conservation laws play a very important role in the solution process and the reduction of PDEs [25-27]. They 
have been used in investigating the existence, uniqueness, and stability of solutions of certain nonlinear PDEs [28-30] and also in the development of numerical methods [31,32].

\section{Traveling Wave Solutions of (3)}

We obtain exact solutions of (3) using Lie group method along with the simplest equation method.

2.1. Non-Topological Soliton Solutions Using Lie Point Symmetries. The vector field

$$
X=\xi^{1} \frac{\partial}{\partial t}+\xi^{2} \frac{\partial}{\partial x}+\xi^{3} \frac{\partial}{\partial y}+\xi^{4} \frac{\partial}{\partial z}+\eta \frac{\partial}{\partial u}
$$

where $\xi^{i}, i=1,2,3,4$ and $\eta$ depend on $t, x, y, z$, and $u$, is a generator of Lie point symmetries of the $(3+1)$-dimensional Boussinesq equation (3) if and only if

$$
\begin{aligned}
& \left.\operatorname{pr}^{(4)} X\left(u_{t t}-u_{x x}-u_{y y}-u_{z z}-\alpha\left(u^{2}\right)_{x x}-u_{x x x x}\right)\right|_{(3)} \\
& \quad=0
\end{aligned}
$$

Here $\operatorname{pr}^{(4)} X$ is the fourth prolongation of the vector field $X$. The invariance condition (5) yields the determining equations, which are a system of linear partial differential equations. Solving this system we obtain the following eight Lie point symmetries:

$$
\begin{aligned}
X_{1}= & \frac{\partial}{\partial x} \\
X_{2}= & \frac{\partial}{\partial t} \\
X_{3}= & \frac{\partial}{\partial y} \\
X_{4}= & \frac{\partial}{\partial z}, \\
X_{5}= & y \frac{\partial}{\partial z}-z \frac{\partial}{\partial y}, \\
X_{6}= & z \frac{\partial}{\partial t}+t \frac{\partial}{\partial z}, \\
X_{7}= & y \frac{\partial}{\partial t}+t \frac{\partial}{\partial y}, \\
X_{8}= & -2 \alpha t \frac{\partial}{\partial t}-\alpha x \frac{\partial}{\partial x}-2 \alpha y \frac{\partial}{\partial y}-2 \alpha z \frac{\partial}{\partial z} \\
& +(1+2 \alpha u) \frac{\partial}{\partial u} .
\end{aligned}
$$

To obtain the nontopological soliton solution of (3), we use the combination of the four translation symmetries, namely, $X=X_{1}+X_{2}+X_{3}+\mu X_{4}$, where $\mu$ is a constant. Solving the associated Lagrange system for $X$, we obtain the four invariants

$$
\begin{gathered}
g=t-x, \quad f=t-y, \\
h=\mu t-z, \quad \theta=u .
\end{gathered}
$$

Now considering $\theta$ as the new dependent variable and $g$, $f$, and $h$ as new independent variables, (3) transforms to a nonlinear PDE in three independent variables, namely,

$$
\begin{gathered}
2 \mu \theta_{f h}+2 \mu \theta_{g h}+2 \theta_{f g}+\left(\mu^{2}-1\right) \theta_{h h} \\
-2 \alpha \theta_{g}^{2}-2 \alpha \theta \theta_{g g}-\theta_{g g g g}=0 .
\end{gathered}
$$

The Lie point symmetries of (8) are

$$
\begin{aligned}
\Gamma_{1}= & \frac{\partial}{\partial g}, \\
\Gamma_{2}= & \frac{\partial}{\partial f}, \\
\Gamma_{3}= & \frac{\partial}{\partial h}, \\
\Gamma_{4}= & \left(2 \alpha f \mu^{3}-4 \alpha h \mu^{2}-2 \alpha f \mu\right) \frac{\partial}{\partial h} \\
& +\left(2 \mu^{2} \alpha f-\alpha \mu^{2} g-\alpha f-3 h \alpha \mu\right) \frac{\partial}{\partial g} \\
& +\left(2 \alpha \mu^{2} \theta+\mu^{2}+1\right) \frac{\partial}{\partial \theta}, \\
\Gamma_{5}= & \left(\alpha f \mu^{3}-\alpha f \mu-3 \alpha h \mu^{2}\right) \frac{\partial}{\partial h} \\
& +\left(\mu^{2} \alpha f-\alpha \mu^{2} g-\alpha f-2 h \alpha \mu\right) \frac{\partial}{\partial g} \\
& -\left(\alpha f \mu^{2}\right) \frac{\partial}{\partial f}+\left(2 \alpha \mu^{2} \theta+\mu^{2}+1\right) \frac{\partial}{\partial \theta} .
\end{aligned}
$$

The use of the combination $\Gamma=\Gamma_{1}+\Gamma_{2}+\beta \Gamma_{3}$, ( $\beta$ is a constant $)$ of the three translation symmetries, gives us the three invariants

$$
r=f-g, \quad w=\beta f-h, \quad \theta=\phi .
$$

Treating $\phi$ as the new dependent variable and $r$ and $w$ as new independent variables, (8) transforms to

$$
\begin{gathered}
\left(\mu^{2}-2 \mu \beta-1\right) \phi_{w w}-2 \beta \phi_{r w}-2 \phi_{r r} \\
-2 \alpha \phi_{r}^{2}-2 \alpha \phi \phi_{r r}-\phi_{r r r}=0
\end{gathered}
$$


which is a nonlinear PDE in two independent variables. Equation (11) has three Lie point symmetries, namely,

$$
\begin{aligned}
\Sigma_{1}= & \frac{\partial}{\partial w}, \\
\Sigma_{2}= & \frac{\partial}{\partial r}, \\
\Sigma_{3}= & \left(4 w \mu \alpha \beta+2 w \alpha-2 w \mu^{2} \alpha\right) \frac{\partial}{\partial w} \\
& +\left(w \alpha \beta+2 \mu r \alpha \beta+r \alpha-\mu^{2} r \alpha\right) \frac{\partial}{\partial r} \\
& +\left(\beta^{2}-4 \mu \beta-2+2 \mu^{2}-4 \alpha \phi \mu \beta-2 \alpha \phi+2 \alpha \mu^{2} \phi\right) \frac{\partial}{\partial \phi},
\end{aligned}
$$

and the symmetry $\Sigma=\Sigma_{1}+\delta \Sigma_{2}$ ( $\delta$ is a constant) provides the two invariants

$$
\xi=\delta w-r, \quad \phi=\psi,
$$

which gives rise to a group invariant solution $\psi=\psi(\xi)$. Using these invariants, the PDE (11) transforms to

$$
\begin{gathered}
\left(\mu^{2} \delta^{2}-2 \mu \beta \delta^{2}-\delta^{2}+2 \beta \delta-2\right) \psi^{\prime \prime} \\
-2 \alpha \psi^{\prime 2}-2 \alpha \psi \psi^{\prime \prime}-\psi^{\prime \prime \prime \prime}=0,
\end{gathered}
$$

which is a fourth-order nonlinear ODE. This ODE can be integrated easily. Integrating it four times while choosing the constants of integration to be zero (because we are looking for soliton solutions) and then reverting back to our original variables $t, x, y, z, u$, we obtain the following group-invariant (nontopological soliton) solutions of the Boussinesq equation (3):

$$
u(x, y, t, z)=\frac{A_{1}}{A_{2}} \operatorname{sech}^{2}\left[\frac{\sqrt{A_{1}}}{2}(B \pm \xi)\right],
$$

where $B$ is a constant of integration and

$$
\begin{aligned}
& A_{1}=\mu^{2} \delta^{2}-2 \mu \beta \delta^{2}-\delta^{2}+2 \beta \delta-2, \\
& A_{2}=\frac{2 \alpha}{3} \\
& \xi=\delta z+(1-\beta \delta) y-x+(\delta \beta-\delta \mu) t
\end{aligned}
$$

2.2. Exact Solutions of (3) Using Simplest Equation Method. We now use the simplest equation method to obtain more solutions of the nonlinear ODE (14), which will then give us more exact solutions for our Boussinesq equation (3). Bernoulli and Riccati equations will be used as the simplest equations $[22,23]$.

2.2.1. Solutions of (3) Using the Bernoulli Equation as the Simplest Equation. In this case the balancing procedure yields $M=2$ so the solutions of (14) have the form

$$
F(k)=A_{0}+A_{1} G+A_{2} G^{2} .
$$

Inserting (17) into (14) and using the Bernoulli equation [23] and then equating the coefficients of powers of $G^{i}$ to zero gives us the following algebraic system of six equations:

$$
\begin{aligned}
& -20 \alpha A_{2}^{2} b^{2}-120 A_{2} b^{4}=0, \\
& -336 A_{2} a b^{3}-24 A_{1} b^{4}-36 \alpha A_{2}^{2} a b \\
& -24 \alpha A_{1} b^{2} A_{2}=0, \\
& -2 A_{1} a^{2}-2 A_{1} a^{2} \delta^{2} \beta \mu-A_{1} a^{4}+2 A_{1} a^{2} \delta \beta \\
& +A_{1} a^{2} \delta^{2} \mu^{2}-\delta^{2} A_{1} a^{2}-2 \alpha A_{0} A_{1} a^{2}=0, \\
& -6 \alpha A_{1}^{2} b^{2}+6 A_{2} b^{2} \delta^{2} \mu^{2}-12 \alpha A_{0} A_{2} b^{2}-6 \delta^{2} A_{2} b^{2} \\
& +12 A_{2} b^{2} \delta \beta-12 A_{2} b^{2}-16 \alpha A_{2}^{2} a^{2} \\
& -12 A_{2} b^{2} \delta^{2} \beta \mu-330 A_{2} a^{2} b^{2} \\
& -42 \alpha A_{1} a A_{2} b-60 A_{1} a b^{3}=0, \\
& -6 A_{1} a b \delta^{2} \beta \mu-16 A_{2} a^{4}-6 \alpha A_{0} A_{1} a b+3 A_{1} a b \delta^{2} \mu^{2} \\
& -8 \alpha A_{0} A_{2} a^{2}-8 A_{2} a^{2} \delta^{2} \beta \mu \\
& -4 \alpha A_{1}^{2} a^{2}-3 \delta^{2} A_{1} a b+6 A_{1} a b \delta \beta-15 A_{1} a^{3} b \\
& -8 A_{2} a^{2}-6 A_{1} a b+4 A_{2} a^{2} \delta^{2} \mu^{2} \\
& -4 \delta^{2} A_{2} a^{2}+8 A_{2} a^{2} \delta \beta=0, \\
& 10 A_{2} a b \delta^{2} \mu^{2}-130 A_{2} a^{3} b-20 A_{2} a b-4 A_{1} b^{2} \delta^{2} \beta \mu \\
& +4 A_{1} b^{2} \delta \beta-4 A_{1} b^{2} \\
& -4 \alpha A_{0} A_{1} b^{2}+20 A_{2} a b \delta \beta-50 A_{1} a^{2} b^{2} \\
& -10 \delta^{2} A_{2} a b-10 \alpha A_{1}^{2} a b-18 \alpha A_{1} a^{2} A_{2} \\
& -2 \delta^{2} A_{1} b^{2}+2 A_{1} b^{2} \delta^{2} \mu^{2}-20 \alpha A_{0} A_{2} a b \\
& -20 A_{2} a b \delta^{2} \beta \mu=0 .
\end{aligned}
$$

These equations can be solved with the aid of Mathematica and one possible solution for $A_{0}, A_{1}$, and $A_{2}$ is

$$
\begin{aligned}
& A_{0}=\frac{-2-2 \delta^{2} \beta \mu-a^{2}+2 \delta \beta+\delta^{2} \mu^{2}-\delta^{2}}{2 \alpha}, \\
& A_{1}=\frac{-6 a b}{\alpha}, \\
& A_{2}=\frac{-6 b^{2}}{\alpha} .
\end{aligned}
$$


Consequently, returning back to the original variables, a solution of (3) is [23]

$$
\begin{aligned}
& u(t, x, y, z) \\
& =A_{0}+A_{1} a\left\{\frac{\cosh [a(\xi+C)]+\sinh [a(\xi+C)]}{1-b \cosh [a(\xi+C)]-b \sinh [a(\xi+C)]}\right\} \\
& \quad+A_{2} a^{2}\left\{\frac{\cosh [a(\xi+C)]+\sinh [a(\xi+C)]}{1-b \cosh [a(\xi+C)]-b \sinh [a(\xi+C)]}\right\}^{2},
\end{aligned}
$$

where $\xi=\delta z+(1-\alpha \delta) y-x+(\delta \beta-\delta \mu) t$ and $C$ is an arbitrary constant of integration.

2.2.2. Solutions of (3) Using the Riccati Equation as the Simplest Equation. Here the balancing procedure gives $M=$ 2 so the solutions of (14) are of the form

$$
F(z)=A_{0}+A_{1} G+A_{2} G^{2}
$$

Substituting (21) into (14) and using the Riccati equation [23], as before, we obtain the following algebraic system of equations in terms of $A_{0}, A_{1}$, and $A_{2}$ :

$$
\begin{gathered}
-20 \alpha A_{2}^{2} b^{2}-120 A_{2} b^{4}=0 \\
-336 A_{2} a b^{3}-24 A_{1} b^{4}-24 \alpha A_{1} b^{2} A_{2} \\
-36 \alpha A_{2}^{2} a b=0 \\
-42 \alpha A_{1} a A_{2} b-6 \delta^{2} A_{2} b^{2}-12 A_{2} b^{2} \\
-330 A_{2} a^{2} b^{2}-6 \alpha A_{1}^{2} b^{2}-16 \alpha A_{2}^{2} a^{2} \\
+12 A_{2} b^{2} \delta \beta-32 \alpha A_{2}^{2} d b+6 A_{2} b^{2} \delta^{2} \mu^{2} \\
-12 A_{2} b^{2} \delta^{2} \beta \mu-240 A_{2} b^{3} d-60 A_{1} a b^{3} \\
-12 \alpha A_{0} A_{2} b^{2}=0, \\
-2 A_{1} a d \delta^{2} \beta \mu-2 \delta^{2} A_{2} d^{2}+2 A_{1} a d \delta \beta \\
-4 A_{2} d^{2}-A_{1} a^{3} d-4 \alpha A_{0} A_{2} d^{2}-2 \alpha A_{1}^{2} d^{2} \\
-2 \alpha A_{0} A_{1} a d-\delta^{2} A_{1} a d-16 A_{2} b d^{3} \\
+2 A_{2} d^{2} \delta^{2} \mu^{2}+4 A_{2} d^{2} \delta \beta-8 A_{1} a b d^{2} \\
-4 A_{2} d^{2} \delta^{2} \beta \mu-2 A_{1} a d+A_{1} a d \delta^{2} \mu^{2} \\
-14 A_{2} a^{2} d^{2}=0,
\end{gathered}
$$

$-20 A_{2} a b \delta^{2} \beta \mu-18 \alpha A_{1} a^{2} A_{2}-36 \alpha A_{1} d A_{2} b$

$-10 \delta^{2} A_{2} a b-20 A_{2} a b-10 \alpha A_{1}^{2} a b$

$-2 \delta^{2} A_{1} b^{2}+4 A_{1} b^{2} \delta \beta-4 A_{1} b^{2} \delta^{2} \beta \mu$

$-4 A_{1} b^{2}-130 A_{2} a^{3} b-440 A_{1} a d b^{2}$

$+10 A_{2} a b \delta^{2} \mu^{2}-28 \alpha A_{2}^{2} d a-4 \alpha A_{0} A_{1} b^{2}$

$-50 A_{1} a^{2} b^{2}+2 A_{1} b^{2} \delta^{2} \mu^{2}-20 \alpha A_{0} A_{2} a b$

$+20 A_{2} a b \delta \beta-40 A_{1} d b^{3}=0$,

$-12 A_{1} d a \delta^{2} \beta \mu-4 A_{1} b d \delta^{2} \beta \mu+2 A_{1} b d \delta^{2} \mu^{2}$

$+6 A_{2} d a \delta^{2} \mu^{2}+4 A_{1} b d \delta \beta+12 A_{2} d a \delta \beta$

$-4 \alpha A_{0} A_{1} b d-12 \alpha A_{0} A_{2} d a-2 A_{1} a^{2} \delta^{2} \beta \mu$

$+A_{1} a^{2} \delta^{2} \mu^{2}+2 A_{1} a^{2} \delta \beta-2 \delta^{2} A_{1} b d-6 \delta^{2} A_{1} d a$

$-6 \alpha A_{1}^{2} d a-12 \alpha A_{1} d^{2} A_{2}-2 \alpha A_{0} A_{1} a^{2}$

$-120 A_{2} a d^{2} b-22 A_{1} a^{2} b d-A_{1} a^{4}$

$-2 A_{1} a^{2}-4 A_{1} b d-\delta^{2} A_{1} a^{2}-12 A_{2} d a$

$-16 A_{1} b^{2} d^{2}-30 A_{2} a^{3} d=0$,

$-16 A_{2} d b \delta^{2} \beta \mu-6 A_{1} a b \delta^{2} \beta \mu+8 A_{2} d b \delta^{2} \mu^{2}$

$-8 A_{2} a^{2} \delta^{2} \beta \mu+6 A_{1} a b \delta \beta+16 A_{2} d b \delta \beta$

$-30 \alpha A_{1} d A_{2} a-6 \alpha A_{0} A_{1} a b-16 \alpha A_{0} A_{2} d b$

$+3 A_{1} a b \delta^{2} \mu^{2}+4 A_{2} a^{2} \delta^{2} \mu^{2}+8 A_{2} a^{2} \delta \beta$

$-3 \delta^{2} A_{1} a b-8 \delta^{2} A_{2} d b-8 \alpha A_{1}^{2} d b-8 \alpha A_{0} A_{2} a^{2}$

$-60 A_{1} a d b^{2}-232 A_{2} a^{2} d b-8 A_{2} a^{2}$

$-16 A_{2} a^{4}-4 \alpha A_{2}^{2} a^{2}-15 A_{1} a^{3} b-16 A_{2} d b$

$-136 A_{2} b^{2} d^{2}-12 \alpha A_{2}^{2} d^{2}-4 \delta^{2} A_{2} a^{2}$

$-6 A_{2} a b=0$.

Solving the above equations yields

$$
\begin{aligned}
& A_{0}=\frac{-a^{2}-8 b d-\delta^{2}-2+2 \delta \beta+\delta^{2} \mu^{2}-2 \delta^{2} \beta \mu}{2 \alpha}, \\
& A_{1}=\frac{-6 a b}{\alpha}, \\
& A_{2}=\frac{-6 b^{2}}{\alpha},
\end{aligned}
$$


and, consequently, the solutions of (3) are

$$
\begin{aligned}
& u(t, x, y, z) \\
& =A_{0}+A_{1}\left\{-\frac{a}{2 b}-\frac{\theta}{2 b} \tanh \left[\frac{1}{2} \theta(\xi+C)\right]\right\} \\
& +A_{2}\left\{-\frac{a}{2 b}-\frac{\theta}{2 b} \tanh \left[\frac{1}{2} \theta(\xi+C)\right]\right\}^{2}, \\
& u(t, x, y, z) \\
& =A_{0} \\
& +A_{1}\left\{-\frac{a}{2 b}-\frac{\theta}{2 b} \tanh \left(\frac{1}{2} \theta \xi\right)\right. \\
& \left.+\frac{\operatorname{sech}(\theta \xi / 2)}{C \cosh (\theta \xi / 2)-(2 b / \theta) \sinh (\theta \xi / 2)}\right\} \\
& +A_{2}\left\{-\frac{a}{2 b}-\frac{\theta}{2 b} \tanh \left(\frac{1}{2} \theta \xi\right)\right. \\
& \left.+\frac{\operatorname{sech}(\theta \xi / 2)}{C \cosh (\theta \xi / 2)-(2 b / \theta) \sinh (\theta \xi / 2)}\right\}^{2},
\end{aligned}
$$

where $\xi=\delta z+(1-\alpha \delta) y-x+(\delta \beta-\delta \mu) t$ and $C$ is an arbitrary constant of integration.

\section{Conservation Laws for (3)}

We utilize the new conservation theorem due to Ibragimov [24] to obtain conservation laws for the $(3+1)$-dimensional Boussinesq equation (3) written as

$$
u_{t t}-u_{x x}-u_{y y}-u_{z z}-2 \alpha u_{x}^{2}-2 \alpha u u_{x x}-u_{x x x x}=0 .
$$

For details of notations, definitions, and theorems the reader is referred to [24].

In Section 2.1 we derived the following eight Lie point symmetries of equation (25):

$$
\begin{aligned}
& X_{1}=\frac{\partial}{\partial x} \\
& X_{2}=\frac{\partial}{\partial t} \\
& X_{3}=\frac{\partial}{\partial y} \\
& X_{4}=\frac{\partial}{\partial z} \\
& X_{5}=y \frac{\partial}{\partial z}-z \frac{\partial}{\partial y},
\end{aligned}
$$

$$
\begin{aligned}
X_{6}= & z \frac{\partial}{\partial t}+t \frac{\partial}{\partial z}, \\
X_{7}= & y \frac{\partial}{\partial t}+t \frac{\partial}{\partial y}, \\
X_{8}= & -2 \alpha t \frac{\partial}{\partial t}-\alpha x \frac{\partial}{\partial x}-2 \alpha y \frac{\partial}{\partial y}-2 \alpha z \frac{\partial}{\partial z} \\
& +(1+2 \alpha u) \frac{\partial}{\partial u} .
\end{aligned}
$$

Corresponding to each of these eight Lie point symmetries we shall construct eight conserved vectors. By definition [24] the adjoint equation of (25) is given by

$$
\begin{gathered}
E^{*}\left(t, x, u, v, \ldots, u_{x x x x}, v_{x x x x}\right) \\
=\frac{\delta}{\delta u}\left[v \left(u_{t t}-u_{x x}-u_{y y}-u_{z z}-2 \alpha u_{x}^{2}\right.\right. \\
\left.\left.-2 \alpha u u_{x x}-u_{x x x x}\right)\right]=0
\end{gathered}
$$

which gives

$$
v_{t t}-v_{x x}-v_{y y}-v_{z z}-2 \alpha u v_{x x}-v_{x x x x}=0 .
$$

Here $v=v(t, x, y, z)$ is a new dependent variable. Clearly, (25) is not self-adjoint. The Lagrangian for the system of (25) and (28) is given by

$$
\begin{gathered}
\mathscr{L}=v\left(u_{t t}-u_{x x}-u_{y y}-u_{z z}-2 \alpha u_{x}^{2}\right. \\
\left.-2 \alpha u u_{x x}-u_{x x x x}\right) .
\end{gathered}
$$

(i) Consider first the translation symmetry $X_{1}=\partial / \partial x$. In this case the operator $Y_{1}[24]$ is the same as $X_{1}$ and the Lie characteristic function $W=-u_{x}$. Thus the components [24] $T^{i}, i=1,2,3,4$, of the conserved vector $T=\left(T^{1}, T^{2}, T^{3}, T^{4}\right)$ are given by

$$
\begin{aligned}
T^{1}= & u_{x} v_{t}-v u_{t x}, \\
T^{2}= & v u_{t t}-v u_{y y}-v u_{z z}-u_{x} v_{x} \\
& -2 \alpha u u_{x} v_{x}-u_{x} v_{x x x}+v_{x x} u_{x x}-v_{x} u_{x x x}, \\
T^{3}= & -u_{x} v_{y}+v u_{x y}, \\
T^{4}= & -u_{x} v_{z}+v u_{x z} .
\end{aligned}
$$

(ii) The second translation symmetry $X_{2}=\partial / \partial t$ gives $W=$ $-u_{t}$. Hence the symmetry generator $X_{2}$ gives rise to the following components of the conserved vector:

$$
\begin{aligned}
T^{1}= & -v u_{x x}-v u_{y y}-v u_{z z}-2 \alpha v u_{x}^{2}-2 \alpha u v u_{x x} \\
& -v u_{x x x x}+u_{t} v_{t}, \\
T^{2}= & 2 \alpha v u_{t} u_{x}-u_{t} v_{x}-2 \alpha u u_{t} v_{x}-u_{t} v_{x x x} \\
& +v u_{t x}+2 \alpha u v u_{t x}+v_{x x} u_{t x}-v_{x} u_{t x x}+v u_{t x x x}, \\
T^{3}= & -v_{y} u_{t}+v u_{t y}, \\
T^{4}= & -v_{z} u_{t}+v u_{t z} .
\end{aligned}
$$


(iii) For the third symmetry $X_{3}=\partial / \partial y$, we have $W=-u_{y}$ and the corresponding components of the conserved vector are

$$
\begin{aligned}
T^{1}= & v_{t} u_{y}-v u_{t y} \\
T^{2}= & -u_{y} v_{x}+2 \alpha v u_{y} u_{x}-2 \alpha u u_{y} v_{x}-u_{y} v_{x x x} \\
& +v u_{x y}+2 \alpha u v u_{x y}+v_{x x} u_{x y}-v_{x} u_{x x y}+v u_{x x x y} \\
T^{3}= & v u_{t t}-v u_{x x}-v u_{z z}-2 \alpha v u_{x}^{2} \\
& -2 \alpha u v u_{x x}-v u_{x x x x}-u_{y} v_{y} \\
T^{4}= & -v_{z} u_{y}+v u_{y z} .
\end{aligned}
$$

(iv) The fourth symmetry $X_{4}=\partial / \partial z$ gives $W=-u_{z}$ and the corresponding components of the conserved vector are

$$
\begin{aligned}
T^{1}= & v_{t} u_{z}-v u_{t z} \\
T^{2}= & -u_{z} v_{x}+2 \alpha v u_{z} u_{x}-2 \alpha u u_{z} v_{x}-u_{z} v_{x x x} \\
& +v u_{x z}+2 \alpha u v u_{x z}+v_{x x} u_{x z}-v_{x} u_{x x z}+v u_{x x x z}, \\
T^{3}= & -v_{y} u_{z}+v u_{y z}, \\
T^{4}= & v u_{t t}-v u_{x x}-v u_{y y}-2 \alpha v u_{x}^{2}-2 \alpha u v u_{x x} \\
& -v u_{x x x x}-u_{z} v_{z} .
\end{aligned}
$$

(v) For the symmetry $X_{5}=y \partial / \partial z-z \partial / \partial y$, we have $W=$ $-y u_{z}+z u_{y}$ and the corresponding components of the conserved vector, as before, are given by

$$
\begin{aligned}
T^{1}= & y u_{z} v_{t}-z u_{y} v_{t}-y v u_{t z}+z v u_{t y}, \\
T^{2}= & 2 \alpha y v u_{x} u_{z}-y u_{z} v_{x}-2 \alpha y u u_{z} v_{x}-y u_{z} v_{x x x} \\
& -4 \alpha z v u_{x} u_{y}+z u_{y} v_{x}+2 \alpha z v u_{y} u_{x} \\
& +2 \alpha z u u_{y} v_{x}+z u_{y} v_{x x x x}+y v u_{x z}+2 \alpha y u v u_{x z} \\
& +y u_{x z} v_{x x}-z v u_{x y}-2 \alpha z u v u_{x y} \\
& -z u_{x y} v_{x x}-y v_{x} u_{x x z}-z v_{x} u_{x x y}-y v u_{x x x z}-z v u_{x x x y}, \\
T^{3}= & -z v u_{t t}+z v u_{x x}+z v u_{z z}+2 \alpha z v u_{x}^{2} \\
& +2 \alpha z u v u_{x x}+z v u_{x x x x}-y v_{y} u_{z}+z v_{y} u_{y} \\
& +v u_{t}+y v u_{y z}+v u_{z}, \\
T^{4}= & y v u_{t t}-z v u_{x x}-z v u_{y y}-2 \alpha y v u_{x}^{2} \\
& -2 \alpha y u v u_{x x}-y v u_{x x x x}-y v_{z} u_{z}+z v_{z} u_{y} \\
& -v u_{y}-z v u_{y z} .
\end{aligned}
$$

(vi) Likewise, the symmetry $X_{6}=z \partial / \partial t+t \partial / \partial z$ gives $W=-z u_{t}-t u_{z}$ and the corresponding components of the conserved vector are given by

$$
\begin{aligned}
T^{1}= & -z v u_{x x}-z v u_{y y}-z v u_{z z}-2 \alpha z v u_{x}^{2} \\
& -2 \alpha z u v u_{x x}-z v u_{x x x x}+z v_{t} u_{t}+t v_{t} u_{z} \\
& -v u_{z}-t v u_{t z}, \\
T^{2}= & 2 \alpha z v u_{x} u_{t}-z u_{t} v_{x}-2 \alpha z u u_{t} v_{x}-z u_{t} v_{x x x} \\
& +2 \alpha t v u_{x} u_{z}-t u_{z} v_{x}-2 \alpha t u u_{z} v_{x} \\
& -t u_{z} v_{x x x}+2 v u_{t x}+2 \alpha z u v u_{t x}+z u_{t x} v_{x x} \\
& +t v u_{x z}+2 \alpha t u v u_{x z} \\
& +t u_{x z} v_{x x}-z v_{x} u_{t x x}-t v_{x} u_{x x z}+z v u_{t x x x} \\
& +t v u_{x x x z}, \\
T^{3}= & -z u_{t} v_{y}-t u_{z} v_{y}+z v u_{t y}+t v u_{y z}, \\
T^{4}= & t v u_{t t}-t v u_{x x}-t v u_{y y}-2 \alpha t v u_{x}^{2} \\
& -2 \alpha t u v u_{x x}-t v u_{x x x x}-z v_{t} u_{z}-t v_{z} u_{z} \\
& +v u_{t}+z v u_{t z} .
\end{aligned}
$$

(vii) As before, the symmetry $X_{7}=y \partial / \partial t+t \partial / \partial y$ yields $W=-y u_{t}-t u_{y}$ and the corresponding components of the conserved vector are given by

$$
\begin{aligned}
T^{1}= & -y v u_{x x}-y v u_{y y}-y v u_{z z}-2 \alpha y v u_{x}^{2} \\
& -2 \alpha y u v u_{x x}-y v u_{x x x x}+y v_{t} u_{t}+t v_{t} u_{y} \\
& -v u_{y}-t v u_{t y}, \\
T^{2}= & 2 \alpha y v u_{x} u_{t}-y u_{t} v_{x}-2 \alpha y u u_{t} v_{x}-y u_{t} v_{x x x} \\
& +4 \alpha t v u_{x} u_{y}-t u_{y} v_{x}-2 \alpha t u u_{y} v_{x} \\
& -2 \alpha t v u_{x} u_{y}-t u_{y} v_{x x x}+y v u_{t x}+2 \alpha y u v u_{t x} \\
& +y u_{t x} v_{x x}+t v u_{x y}+2 \alpha t u v u_{x y} \\
& +t u_{x y} v_{x x}-y v_{x} u_{t x x}-t v_{x} u_{x x y}+y v u_{t x x x} \\
& +t v u_{x x x y}, \\
T^{3}= & t v u_{t t}-t v u_{x x}-t v u_{y y}-2 \alpha t v u_{x}^{2} \\
& -2 \alpha t u v u_{x x}-t v u_{x x x x}-y v_{y} u_{t}-t v_{y} u_{y} \\
& +v u_{t}+y v u_{t y}, \\
T^{4}= & -y u_{t} v_{z}-t u_{y} v_{z}+y v u_{t z}+t v u_{y z} .
\end{aligned}
$$


(viii) Finally, for the symmetry

$$
\begin{aligned}
X_{8}= & -2 \alpha t \frac{\partial}{\partial t}-x \alpha \frac{\partial}{\partial x}-2 \alpha y \frac{\partial}{\partial y}-2 \alpha z \frac{\partial}{\partial z} \\
& +(1+2 \alpha u) \frac{\partial}{\partial u}
\end{aligned}
$$

the value of $Y_{8}$ is not the same as $X_{8}$ and in fact is given by

$$
\begin{aligned}
Y_{8}= & -2 \alpha t \frac{\partial}{\partial t}-x \alpha \frac{\partial}{\partial x}-2 \alpha y \frac{\partial}{\partial y}-2 \alpha z \frac{\partial}{\partial z} \\
& +(1+2 \alpha u) \frac{\partial}{\partial u}+\alpha v \frac{\partial}{\partial v} .
\end{aligned}
$$

The Lie characteristic function $W=1+2 \alpha u+2 \alpha t u_{t}+x \alpha u_{x}+$ $2 \alpha y u_{y}+2 \alpha z u_{z}$ and, consequently, the conserved vector $T$ has components given by

$$
\begin{aligned}
T^{1}= & 2 \alpha t v u_{x x}+2 \alpha t v u_{y y}+2 \alpha t v u_{z z}+4 \alpha^{2} t v u_{x}^{2} \\
& +4 \alpha^{2} t v u u_{x x}+2 \alpha t v u_{x x x x}-v_{t} \\
& -2 \alpha u v_{t}-2 \alpha t u_{t} v_{t}-\alpha x u_{x} v_{t}-2 \alpha y u_{y} v_{t} \\
& -2 \alpha z u_{z} v_{t}+4 \alpha v u_{t}+\alpha x v u_{t x} \\
& +2 \alpha y v u_{t y}+2 \alpha z v u_{t z}, \\
T^{2}= & -\alpha x v u_{t t}+\alpha x v u_{y y}+\alpha x v u_{z z}-3 \alpha v u_{x} \\
& +v_{x}+4 \alpha u v_{x}+v_{x x x}-8 \alpha^{2} u v u_{x} \\
& +4 \alpha^{2} u^{2} v_{x}+2 \alpha u v_{x x x}+2 \alpha t u_{t} v_{x}-4 \alpha^{2} t v u_{t} u_{x} \\
& +4 \alpha^{2} t u u_{t} v_{x}+2 \alpha t u_{t} v_{x x x} \\
& +\alpha x u_{x} v_{x}+2 \alpha^{2} x u u_{x} v_{x}+\alpha x u_{x} v_{x x x} \\
& +2 \alpha y u_{y} v_{x}-4 \alpha^{2} y v u_{x} u_{y}+4 \alpha^{2} y u u_{y} v_{x} \\
& +2 \alpha y v u_{x x x y}-2 \alpha z v u_{x x x z}, \\
& +2 \alpha y u_{y} v_{x x x}+2 \alpha z u_{z} v_{x}-4 \alpha^{2} z v u_{x} u_{z} \\
& +4 \alpha^{2} z u u_{z} v_{x}+2 \alpha z u_{z} v_{x x x}-2 \alpha t v u_{t x} \\
& -4 \alpha^{2} t u v u_{t x}-2 \alpha t u_{t x} v_{x x}-2 \alpha^{2} u v u_{x} \\
& -3 \alpha u_{x} v_{x x}-\alpha x u_{x x} v_{x x}-2 \alpha y v u_{x y} \\
& -4 \alpha^{2} y u v u_{x y}-2 \alpha y u_{x y} v_{x x}-2 \alpha z v u_{x z} \\
& -4 \alpha^{2} z u v u_{x z}-2 \alpha z u_{x z} v_{x x}+4 \alpha v_{x} u_{x x} \\
& 2 \alpha t v_{x} u_{t x x}+\alpha x v_{x} u_{x x x}+2 \alpha y v_{x} u_{x x y} \\
& 2 \alpha t v u_{t x x x}-5 \alpha v u_{x x x x} \\
&
\end{aligned}
$$

$$
\begin{aligned}
T^{3}= & -2 \alpha y v u_{t t}+2 \alpha y v u_{x x}+2 \alpha y v u_{z z} \\
& +4 \alpha^{2} y v u_{x}^{2}+4 \alpha^{2} y v u u_{x x}+2 \alpha y v u_{x x x x}+v_{y} \\
& +2 \alpha u v_{y}+2 \alpha t u_{t} v_{y}+\alpha x u_{x} v_{y}+2 \alpha y u_{y} v_{y} \\
& +2 \alpha z u_{z} v_{y}-4 \alpha v u_{y}-2 \alpha t v u_{t y} \\
& -\alpha x v u_{x y}-2 \alpha z v u_{z}, \\
T^{4}= & -2 \alpha z v u_{t t}+2 \alpha z v u_{x x}+2 \alpha z v u_{y y} \\
& +4 \alpha^{2} z v u_{x}^{2}+4 \alpha^{2} z v u u_{x x}+2 \alpha z v u_{x x x x}+v_{z} \\
& +2 \alpha u v_{z}+2 \alpha t u_{t} v_{z}+\alpha x u_{x} v_{z}+2 \alpha y u_{y} v_{z} \\
& +2 \alpha z u_{z} v_{z}-4 \alpha v u_{z}-2 \alpha t v u_{t z} \\
& -\alpha x v u_{x z}-2 \alpha y v u_{y z} .
\end{aligned}
$$

Remark. Each conserved vector $T$ obtained above contains the arbitrary solution $v$ of the adjoint equation (28) and hence gives an infinite number of conservation laws.

\section{Conclusions}

Exact solutions of the $(3+1)$-dimensional Boussinesq equation (3) were obtained with the aid of Lie point symmetries of (3) as well as the simplest equation method. The solutions obtained were solitary waves and nontopological soliton. Furthermore, the conservation laws for the $(3+1)$-dimensional Boussinesq equation were also constructed by utilizing the new conservation theorem due to Ibragimov [24].

\section{Conflict of Interests}

The authors declare that there is no conflict of interests regarding the publication of this paper.

\section{Acknowledgment}

Chaudry Masood Khalique would like to thank the NorthWest University, Mafikeng Campus, for its continued support.

\section{References}

[1] J. Boussinesq, “Thorie des Ondes et Des Remous Qui se Propagent le Long d'un Canal Rectangulaire Horizontal, en Communiquant au Liquidecontenudansce Canal des Uitessessensiblementpareilles de la Surface au Fond," Journal de Mathématiques Pures et Appliquées, vol. 17, pp. 55-158, 1872.

[2] G. B. Whitham, Linear and Nonlinear Waves, Wiley, New York, NY, USA, 1974.

[3] Y. Chen, Z. Yan, and H. Zhang, "New explicit solitary wave solutions for $\$(2+1) \$$-dimensional Boussinesq equation," Physics Letters A, vol. 307, no. 2-3, pp. 107-113, 2003.

[4] C. Liu and Z. Dai, "Exact periodic solitary wave solutions for the $(2+1)$-dimensional Boussinesq equation," Journal of 
Mathematical Analysis and Applications, vol.367, no. 2, pp. 444450, 2010.

[5] A. M. Wazwaz, "Non-integrable variants of Boussinesq equation with two solitons," Applied Mathematics and Computation, vol. 217, no. 2, pp. 820-825, 2010.

[6] G. Imed and B. Abderrahmen, "Numerical solution of the (2+1)-dimensional Boussinesq equation with Initial Condition by Homotopy Perturbation Method," Applied Mathematical Sciences, vol. 6, pp. 5993-6002, 2012.

[7] L. D. Moleleki and C. M. Khalique, "Solutions and conservation laws of a (2+1)-dimensional Boussinesq equation," Abstract and Applied Analysis, vol. 2013, Article ID 548975, 8 pages, 2013.

[8] W. Yong-Qi, "Periodic wave solution to the $(3+1)$-dimensional Boussinesq equation," Chinese Physics Letters, vol. 25, no. 8, pp. 2739-2742, 2008.

[9] M. S. Bruzon and M. L. Gandarias, "Symmetries for a family of Boussinesq equations with nonlinear dispersion," Communications in Nonlinear Science and Numerical Simulation, vol. 14, no. 8, pp. 3250-3257, 2009.

[10] M. S. Bruzon, "Exact solutions for a generalized Boussinesq equation," Theoretical and Mathematical Physics, vol. 159, no. 3, pp. 778-785, 2009.

[11] M. L. Gandarias and M. S. Bruzon, "Classical and nonclassical symmetries of a generalized Boussinesq equation," Journal of Nonlinear Mathematical Physics, vol. 5, no. 1, pp. 8-12, 1998.

[12] B. Muatjetjeja and C. M. Khalique, "Conservation laws for a variable coefficient variant Boussinesq system," Abstract and Applied Analysis, vol. 2014, Article ID 169694, 5 pages, 2014.

[13] M. J. Ablowitz and P. A. Clarkson, Solitons, Nonlinear Evolution Equations and Inverse Scattering, Cambridge University Press, Cambridge, UK, 1991.

[14] C. Gu, H. Hu, and Z. Zhou, Darboux Transformation in Soliton Theory and Its Geometric Applications, Springer, Dordrecht, The Netherlands, 2005.

[15] A. Wazwaz, "The tanh and the sine-cosine methods for compact and noncompact solutions of the nonlinear Klein-Gordon equation," Applied Mathematics and Computation, vol. 167, no. 2, pp. 1179-1195, 2005.

[16] R. Hirota, The Direct Method in Soliton Theory, Cambridge University Press, Cambridge, UK, 2004.

[17] S. Liu, Z. Fu, and Q. Zhao, "Jacobi elliptic function expansion method and periodic wave solutions of nonlinear wave equations," Physics Letters A, vol. 289, no. 1-2, pp. 69-74, 2001.

[18] P. J. Olver, Applications of Lie Groups to Differential Equations, vol. 107 of Graduate Texts in Mathematics, Springer, Berlin, Germany, 2nd edition, 1993.

[19] N. H. Ibragimov, CRC Handbook of Lie Group Analysis of Differential Equations, vol. 1, CRC Press, Boca Raton, Fla, USA, 1994.

[20] C. M. Khalique and A. R. Adem, "Exact solutions of a generalized (3+1)-dimensional Kadomtsev-Petviashvili equation using Lie symmetry analysis," Applied Mathematics and Computation, vol. 216, no. 10, pp. 2849-2854, 2010.

[21] J. H. He and X. H. Wu, "Exp-function method for nonlinear wave equations," Chaos, Solitons \& Fractals, vol. 30, no. 3, pp. 700-708, 2006.

[22] N. A. Kudryashov, "Simplest equation method to look for exact solutions of nonlinear differential equations," Chaos, Solitons and Fractals, vol. 24, no. 5, pp. 1217-1231, 2005.

[23] C. M. Khalique, "On the solutions and conservation laws of the $(1+1)$-dimensional higher-order Broer-Kaup system," Boundary Value Problems, vol. 2013, article 41, 18 pages, 2013.
[24] N. H. Ibragimov, "A new conservation theorem," Journal of Mathematical Analysis and Applications, vol. 333, no. 1, pp. 311328, 2007.

[25] A. Sjöberg, "Double reduction of PDEs from the association of symmetries with conservation laws with applications," Applied Mathematics and Computation, vol. 184, no. 2, pp. 608-616, 2007.

[26] A. H. Bokhari, A. Y. Al-Dweik, A. H. Kara, F. M. Mahomed, and F. D. Zaman, "Double reduction of a nonlinear $(2+1)$ wave equation via conservation laws," Communications in Nonlinear Science and Numerical Simulation, vol. 16, no. 3, pp. 1244-1253, 2011.

[27] G. L. Caraffini and M. Galvani, "Symmetries and exact solutions via conservation laws for some partial differential equations of mathematical physics," Applied Mathematics and Computation, vol. 219, no. 4, pp. 1474-1484, 2012.

[28] P. D. Lax, "Integrals of nonlinear equations of evolution and solitary waves," Communications on Pure and Applied Mathematics, vol. 21, pp. 467-490, 1968.

[29] T. B. Benjamin, “The stability of solitary waves," Proceedings of the Royal Society A, vol. 328, pp. 153-183, 1972.

[30] R. J. Knops and C. A. Stuart, "Quasiconvexity and uniqueness of equilibrium solutions in nonlinear elasticity," Archive for Rational Mechanics and Analysis, vol. 86, no. 3, pp. 233-249, 1984.

[31] R. J. LeVeque, Numerical Methods for Conservation Laws, Birkhuser, Basel, Switzerland, 1992.

[32] E. Godlewski and P.-A. Raviart, Numerical Approximation of Hyperbolic Systems of Conservation Laws, vol. 118 of Applied Mathematical Sciences, Springer, Berlin, Germany, 1996. 


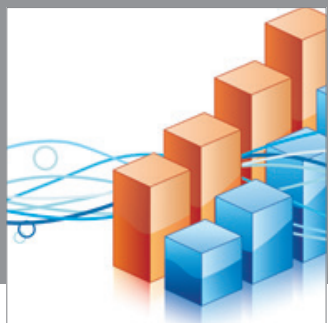

Advances in

Operations Research

mansans

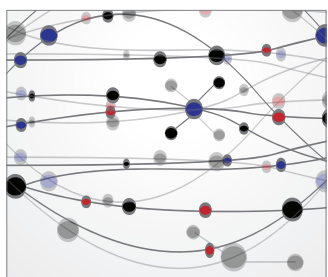

The Scientific World Journal
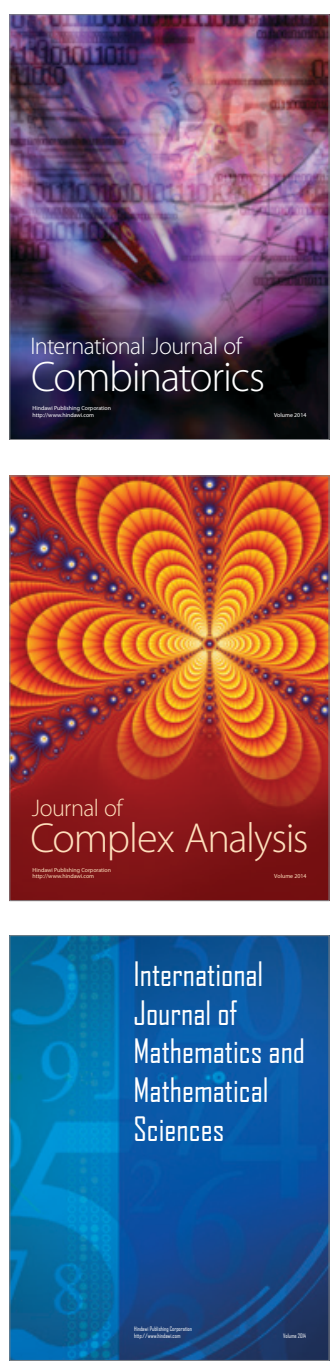
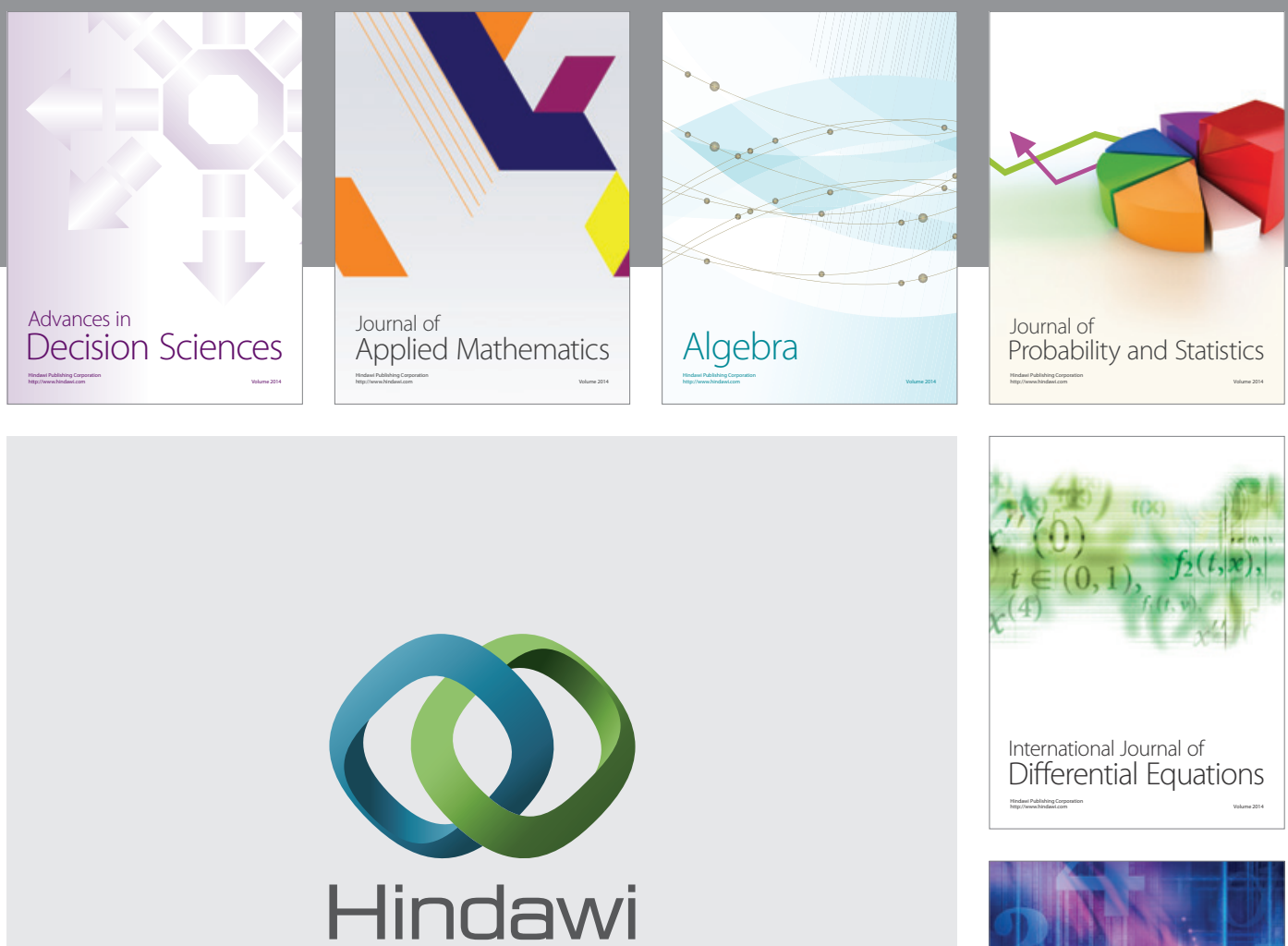

Submit your manuscripts at http://www.hindawi.com
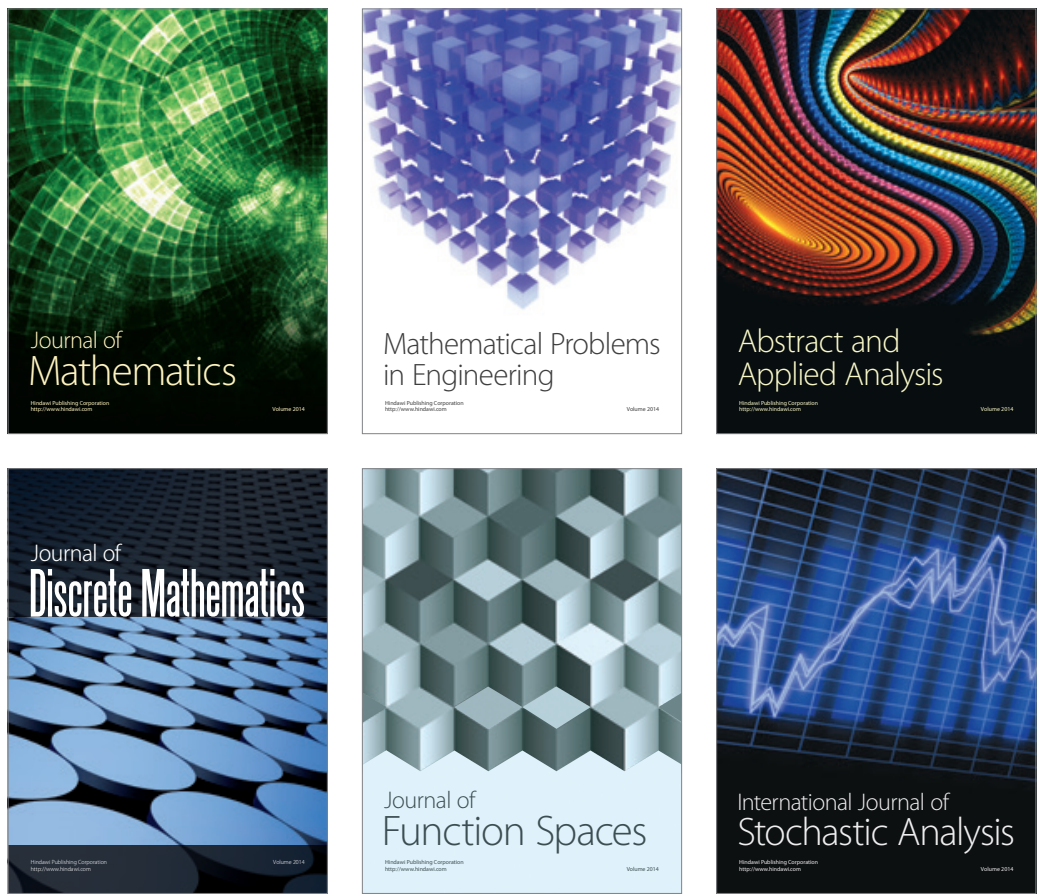

Journal of

Function Spaces

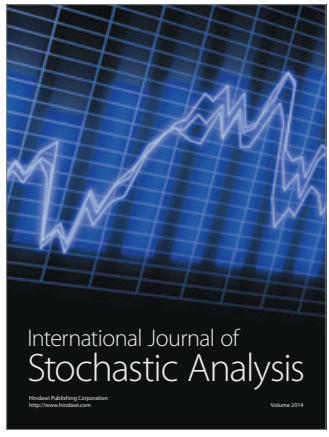

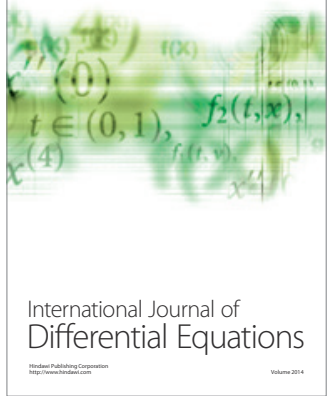
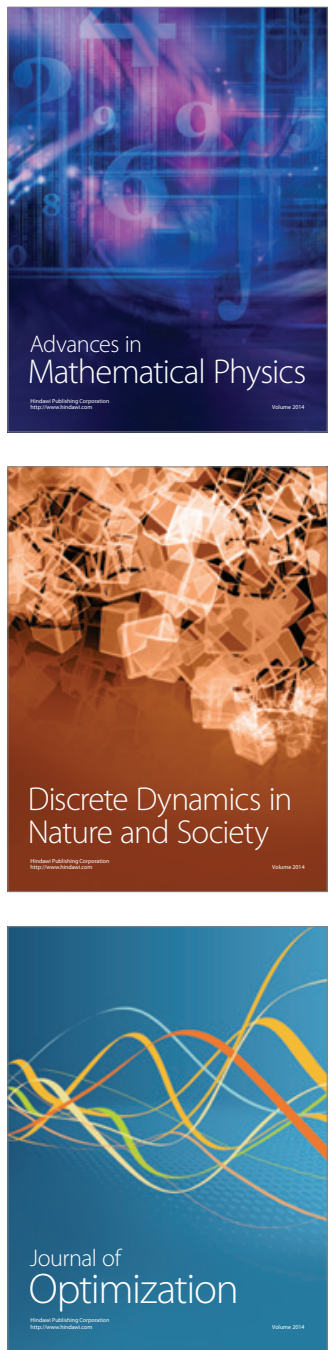